\title{
Shape localization, quantification and correspondence using Region Matching Algorithm
}

\author{
Faraz Janan*1, Sir Michael Brady ${ }^{2}$ \\ ${ }^{1}$ Institute of Bio-Medical Engineering (IBME), University of Oxford, UK \\ ${ }^{2}$ Department of Oncology, University of Oxford, UK
}

Received: January 5, 2014

DOI: $10.5430 /$ jbgc.v6n $1 \mathrm{p} 8$
Accepted: October 27, $2015 \quad$ Online Published: November 24, 2015

URL: http://dx.doi.org/10.5430/jbgc.v6n1p8

\begin{abstract}
We propose a method for local, region-based matching of planar shapes, especially as those shapes that change over time. This is a problem fundamental to medical imaging, specifically the comparison over time of mammograms. The method is based on the non-emergence and non-enhancement of maxima, as well as the causality principle of integral invariant scale space. The core idea of our Region Matching Algorithm (RMA) is to divide a shape into a number of "salient" regions and then to compare all such regions for local similarity in order to quantitatively identify new growths or partial/complete occlusions. The algorithm has several advantages over commonly used methods for shape comparison of segmented regions. First, it provides improved key-point alignment for optimal shape correspondence. Second, it identifies localized changes such as new growths as well as complete/partial occlusion in corresponding regions by dividing the segmented region into sub-regions based upon the extrema that persist over a sufficient range of scales. Third, the algorithm does not depend upon the spatial locations of mammographic features and eliminates the need for registration to identify salient changes over time. Finally, the algorithm is fast to compute and requires no human intervention. We apply the method to temporal pairs of mammograms in order to detect potentially important differences between them.
\end{abstract}

Key Words: Region matching, Shape matching, Occluded shapes, Aligning shapes, Temporal changes, Mammograms

\section{INTRODUCTION}

Shape matching is a key issue and an established area of research in computer vision. In our previous work we have noted that shape description using Integral Invariants (II) yields signatures that are invariant to articulations and bending, and which are robust to noise. Geometric invariants, which are invariant under affine, Euclidean, or similarity transformations, may be used to describe shapes. Shape matching is a particularly challenging problem in medical image analysis, most often for pairs of images of the same patient taken at different times. Such image pairs may show differences that are the result of illness, response to therapy, or, more prosaically differences in the imaging conditions. Projective invariants may help with many small changes; but cannot deal with complete or partial occlusion of articulations of the shape which are frequently diagnostically important to take into account. For example, irregular change in the sizes of nuclei in Fine Needle Aspiration (FNA) may suggest the presence of malignancy. Image registration techniques have previously been used to address this issue..$^{[1,2]}$ Registration techniques, both rigid and non-rigid, yield a dense warp map that establishes correspondences between all pixels inside a shape and focuses on shape retrieval and matching instead of quantifying regions at a local level. Other tech-

*Correspondence: Faraz Janan; Email: farazjanan@yahoo.com; Address: Institute of Bio-Medical Engineering (IBME), University of Oxford, UK. 
niques require a "shape space", which depend on training data to enable comparisons. ${ }^{[3]}$ These approaches aside, there appears to have been little or no research aimed at identifying and quantifying new growths and partial occlusions by comparing two planar shapes regardless of scale, spatial variations, and orientation. This is the problem that we address.

The first step in comparing two shapes is to align them by identifying key points. The alignment of curves and matching of shapes is discussed in Ref. ${ }^{[4]}$ based on local curvature information, and often produces good results. However, curvature, being a second derivative, is intrinsically susceptible to noise. Our algorithm imposes a metric based on circular $\mathrm{II}^{[5]}$ on a shape, which, in combination with a scale space analysis, ${ }^{[6]}$ enables us to obtain descriptive information and key points, then divides the shape into a number of regions based on those key points. II are used for shape matching in Ref. ${ }^{[5]}$ In that work, dynamic programing and Fast Marching Algorithms are used to match shapes and to establish point-wise correspondences between them. Those studies neither detect nor quantify changes in shapes. This paper presents a method that does not depend upon a (computationally expensive) optimization algorithm. Instead, we compare shapes as closed planar contours with no self-interactions between them. We use circular II to describe the shapes. II are descriptors that robustly define edges, corners and peaks, thus delineating salient regions in the shapes that we use to subdivide them, again using II scale space.

\section{Background}

We assume that a shape describes a single entity in the form of a closed contour. ${ }^{[3-9]}$ Shape description defines an anatomical structure at several scales of observations. A comprehensive overview of shape representation with respect to application categories is given in Refs. ${ }^{[6,9]}$ A brief list is recalled in Figure 1.

Contour-based shape descriptions are reported extensively in Refs. ${ }^{[4,5]}$ For example, B-splines have been used to represent shapes and to match them. ${ }^{[10-15]}$ Chain codes are an effective shape representation, especially for coding; ${ }^{[16]}$ however, as they are sensitive to discretization errors in rotation and scale, they are rarely used to match shapes. The most widely used methods to describe shapes are based on curvature. ${ }^{[17,18]}$ This can work well and also has an extensive basis in mathematics. ${ }^{[9,19,20]}$ In practice, the main disadvantage of using curvature, which is a differential invariant, is that its measurement is highly sensitive to noise. II, which are fundamentally related to differential invariants, can be used effectively for shape representation ${ }^{[21]}$ and reconstruction, ${ }^{[22]}$ and are robust to noise. ${ }^{[21,23,24]}$ Al-Kadi et al. also measured Published by Sciedu Press feature robustness under noise presence in medical images and is reported in Refs. ${ }^{[25-27]}$ II outperform differential invariants $^{[28,29]}$ for invariance to small perturbations in shapes. Circular II is a structural approach that describes a shape in a unique way, ${ }^{[30]}$ as do conic II. ${ }^{[22]}$ Structural invariants are known to deal with partial occlusions and partial matching of shapes, which further emphasizes their importance in medical imaging.

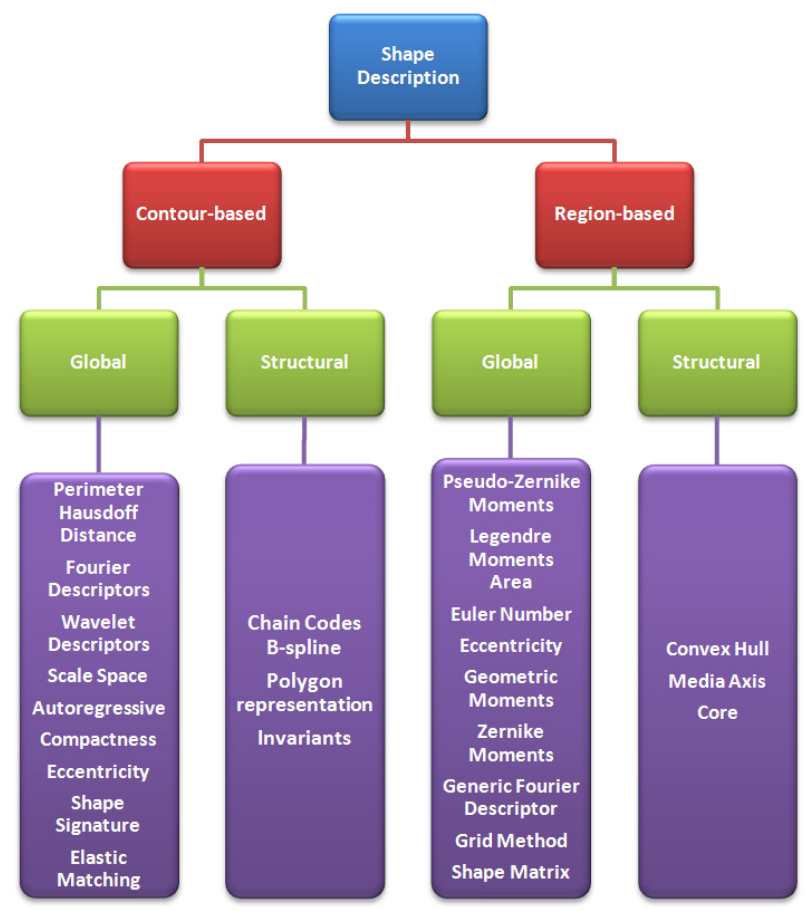

Figure 1. A list of the most popular shape analysis methods proposed to date, together with their classification (mostly described in Ref. ${ }^{[6]}$ )

\section{MeTHODS}

Various methods are discussed briefly to cover the breath of the subject, which relates to a huge body of literature that further amounts to variety applications. As mentioned earlier our method is based on the II scale space, which is inspired from Ref. ${ }^{[24]}$ and eventually provides the basis for the RMA that we develop.

\subsection{Approaches to match shapes}

Typically, point-wise correspondences are established to match shapes based on intrinsic statistical properties found by anatomical modelling or by computing a matching cost for the points on the boundary. ${ }^{[31,32]}$ Landmarks are generally identified in two shapes using a certain descriptor, ${ }^{[33]}$ for example with genetic algorithms, ${ }^{[34]}$ Eccentricity transformations, ${ }^{[35]}$ phase congruency, ${ }^{[36,37]}$ differential methods ${ }^{[17,18,38]}$ or II. Descriptors that represent a shape as a transformation, such as Fourier components, ${ }^{[39]}$ highlight salient 
features in a shape at the cost of suppressing deformation and ignoring translation or rotation. ${ }^{[35]}$ Techniques $^{[36,40-44]}$ that establish a dense correspondence between shapes by embedding $2 \mathrm{D}$ or $3 \mathrm{D}$ shapes in a canonical domain usually fail to deal effectively with shape articulations. Indeed, the fact that they preserve geodesic distances, ${ }^{[45-47]}$ phase angles and other structural features, makes it difficult to cope with isometric deformations, such as bending. ${ }^{[48,49]}$ Other techniques involve shape appearance in descriptions, such as shape skeletons, ${ }^{[50]}$ graph matching, ${ }^{[51-54]}$ contour flexibility, ${ }^{[55]}$ Laplace spectra, ${ }^{[56]}$ partial differential equations ${ }^{[57,58]}$ and the rolling penetrate descriptor. ${ }^{[58]}$ Histogram geometry ${ }^{[56]}$ has also been evaluated to subdivide shapes into parts using topographic features and then to register them.

Sebastian ${ }^{[59,82]}$ presented an alignment criterion which compares one curve to another, one of which is considered to be the model curve. Dijkstra's algorithm was used with encouraging results to correspond points on the two shapes. ${ }^{[18]}$ Other studies ${ }^{[60,61]}$ confirmed its efficiency in matching visual parts and shape contexts. However, the method suffers from the "city block" problem and sub-pixel accuracy, which has subsequently been improved ${ }^{[62]}$ using the Fast Marching Algorithm. ${ }^{[63-65]}$ Other effective methods to match shapes include: Eccentricity transforms, ${ }^{[35,66-68]}$ Skeletonization, ${ }^{[69,70]}$ dynamic programing, ${ }^{[32]}$ Fast Sweeping Algorithm, ${ }^{[71,72]}$ Ant Colony Optimization, ${ }^{[73]}$ Bee Colony optimization, ${ }^{[74-76]}$ and Bending invariants. ${ }^{[77,78]}$ Comprehensive surveys of shape matching techniques with respect to local correspondence can be found in Refs. ${ }^{[79-81]}$

A descriptor is called an invariant if it can accommodate a certain shape transformation class. ${ }^{[82]}$ A projective transformation, from the set of transformations known as the projective group, is widely used in computer vision. Transformation groups or group actions are tools to generate application-specific invariants ${ }^{[36,83-91]}$ and are central to invariant theory. ${ }^{[9,92]}$ Invariants can be algebraic [82,93-95] $^{\left[{ }^{2}\right.}$ geometrical $^{[83,96]}$ combinations of coplanar points or planes; $;^{[94,95,97-103]}$ differential ${ }^{[20,36,104-110]}$ or Integral. ${ }^{[21,29,111,112]}$ Since algebraic and geometric invariants are defined for the whole shape rather than boundary, and since differential invariants are very sensitive to boundary noise, we use circular II that are relatively robust to boundary noise. In this paper, we refer to circular II simply as II. At first glance, II are similar to the SUSAN feature detector, ${ }^{[13]}$ which has been used in a variety of applications ${ }^{[114-120]}$ and reproduced with various enhancements. ${ }^{[120-123]}$ However, the fundamental limitation of SUSAN, which renders it far less relevant in our application, is the assumption of homogeneity in a circular region. Medical images, especially mammograms, are at best piecewise homogeneous. Various shape signatures have been proposed; ${ }^{[21,30,93,112,124,125]}$ however, none of them quantify changes in regions while matching shapes. This is what distinguishes our method.

\subsection{Circular II}

Many et al. ${ }^{[24]}$ applied circular II for shape matching. They showed that it can robustly handle occluded shapes. Despite its simplicity, it gives a unique shape description, which we have previously exploited for region matching and shape correspondence. It has properties of non-emergence and nonenhancement of extrema in a linear fashion. It has also been used for image enhancement and noise suppression. ${ }^{[126]}$ II is similar to Gaussian smoothing in terms of implementation, though it has very different diffusion and related properties.

II is defined ${ }^{[24]}$ by considering a disc $\mathrm{B}_{r}(\mathrm{p})$ of radius $r$ applied to every point $\mathrm{p}$ of a closed contour $\gamma$, parameterized to $\mathrm{n}$ points. The representative function that evaluates the intersection of the disc $\mathrm{B}_{r}(\mathrm{p})$ and the contour $\gamma$ is as follows:

$$
\chi\left(B_{r}(p), C\right)(x)=\left\{\begin{array}{lll}
1 & \text { if } & x \epsilon\left\{B_{r}(p) \cap \bar{\gamma}\right\} \\
0 & \text { otherwise }
\end{array}\right.
$$

Where $\bar{\gamma}$ is the interior of the curve. The local integral area $\mathbf{I}_{r}(\gamma)$ of the curve is given by the function $\mathbf{I}_{\gamma}^{r}(\mathrm{p})$ at every point $\mathrm{p} \epsilon \bar{\gamma}$ with integral kernel $\chi$ as follows:

$$
I_{\gamma}^{r}(p)=\int_{B_{r}(p) \cap \bar{\gamma}}^{n} d x
$$

The range of $\mathrm{I}_{\gamma}^{r}(\mathrm{p})$, is a positive real and spans the interval from zero to the area of the curve bounded by the disc.

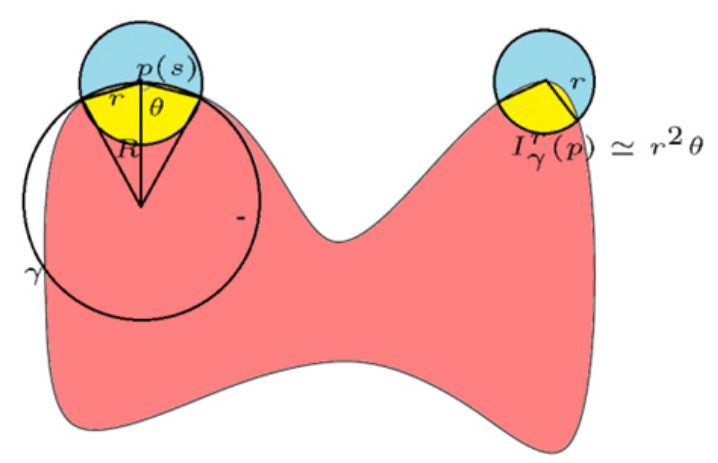

Figure 2. Integral Invariant defined in Eq-2 and explained in Ref. ${ }^{[24]}$

In the above equation $\Omega$ is the domain of the curve C. Figure 2 illustrates II as discussed in Ref. ${ }^{[21]}$ and given by Equation 2. Changing the size of the II kernel creates a scale space, and which does not amplify noise. Rather it suppresses noise; however, at the cost of image details, which is an inevitable consequence of smoothing. In Figure 3, the value of the II for 
shape description occurs when the circle is centered not on a point along the curve but near to it, so that the circle overlaps the pink region. II have a strong and unique expression for encoding shapes and is closely related to curvature functions. In one sense, it is a weighted reciprocal of curvature, where the II shape description is almost a vertically flipped version of the graph generated by curvature. However, curvature

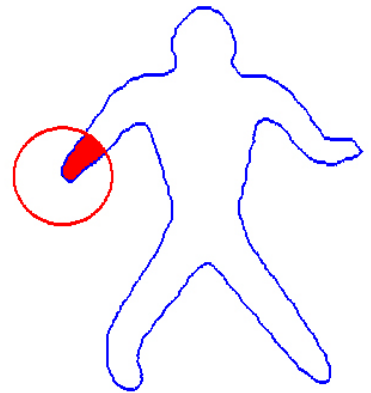

a

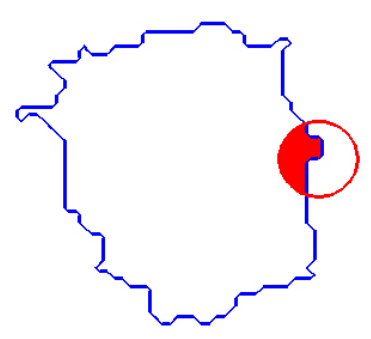

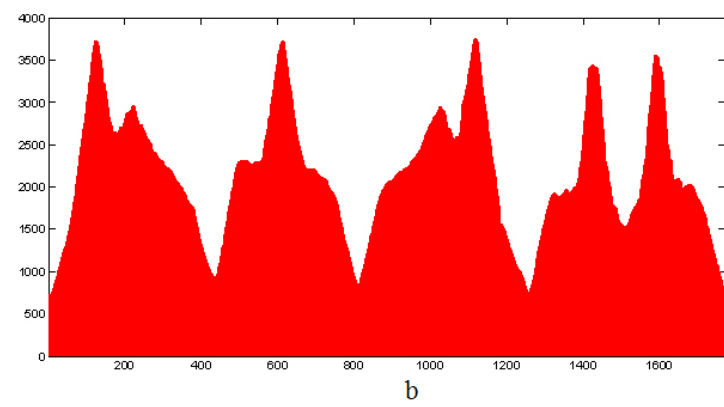

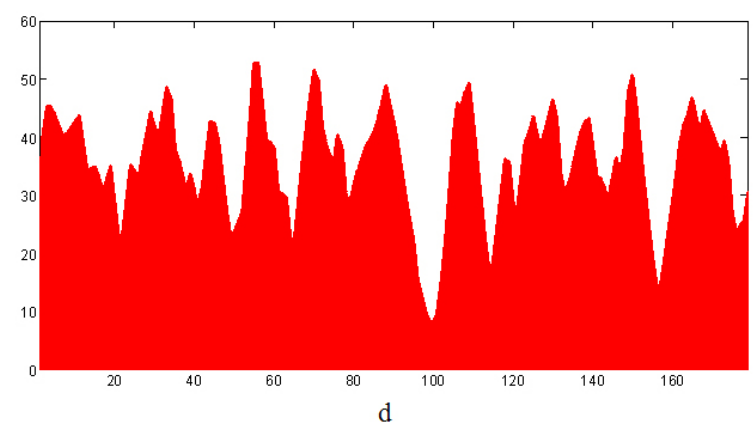

Figure 3. (a) and (c) are two examples of closed polygons with integration kernels imposed on them and highlight the integration area in red; (b) and (d) are the corresponding Integral Invariant for the complete curves. (c) is the outline of a segmented tumour mass from the Mini-MIAS mammographic database $\mathrm{e}^{[127]}$

\subsection{Region Matching Algorithm (RMA)}

This section explains the RMA that we have developed to divide shapes into regions based on the causality of II scale space and then quantifies those regions to estimate articulations and new growth. Though RMA does not require initial alignment for region matching, it can be used to find points of initial alignment that are then used to establish point-wise correspondences of shapes. Currently, regions are matched simply on the basis of a least sum of squared differences, which we have found works well in our application. If the two shapes being compared are not expected to differ substantially, then the normalized difference of centroids of regions can also be used as an additional feature for finding similarity. The variation between two regions can be presented in terms of the number of pixels and percentage difference. The areas of regions might be useful in applications where most of the regions in corresponding shapes are likely to be the similar in size. Other factors such as texture and gradient measures as well as additional shape features can be used with the existing shape descriptor to meet the requirements of a specific application. ${ }^{[127]}$

The core issue in scale space is scale selection. In our case, the maximum scale is selected in accordance with the size of the shape and the size of the II kernel; and is application dependent. The scale space of the kernel should range from being able to identify localized changes in shapes to describing the overall structure; that is, moving from a fine to a coarse scale. At a coarse scale, shape description is less sensitive to small perturbations and noise.

To divide a shape into regions, we first need to set the maximum or coarsest scale. The coarsest scale may be determined automatically by taking the mean value of the shapeto-integration kernel ratio (SIR) of the two shapes. Let $r_{\max }$ be the maximum scale indicator (this equates to the radius of the circular II disc at the maximum scale). Then comparing shapes $\left(S_{1}, S_{2}\right)$ for region matching where the area of SIR can be adjusted depending upon the size and variability of the shapes.

$$
r_{\max }=\left\lceil\operatorname{mean}\left(r_{S_{1 \max }}, r_{S_{2 \max }}\right)\right\rceil
$$

Where $r_{S_{\text {imax }}}=\sqrt{\frac{\text { Areaof } S_{i}}{\operatorname{SIR} * \pi}}, \mathrm{i}=[1,2]$. 


\section{RESULTS}

First, we illustrate the application of RMA to the pair of shapes shown in Figure 4. Figure 5 shows the II signature at the coarsest scale. This illustrates the effectiveness of RMA for initial alignment of two shapes. It finds the best region with the minimum matching cost and designates its starting point as a point of initial alignment. A second example is given in Figure 6, where a new growth has been identified (which corresponds, in fact, to the rabbit's tail). Other examples are given in Figures 7 and 8. The accuracy of initial alignment for shapes with locally distinct geometry is high, as is evident from Table 1.

We have applied RMA to a range of shapes from the Kimia database, and assessed its performance for within-group similarity. The accuracy of region matching is assessed manually as a surrogate for ground truth. Experiments were carried out on 146 dissimilar shapes from four similar object groups of the Kimia database. Region matching for each pair of shapes was assigned a score from 1-6, whereas the accuracy of new growth and occluded regions was assessed qualitatively and assigned a grade from 1-4. The results are summarized in Table 1.
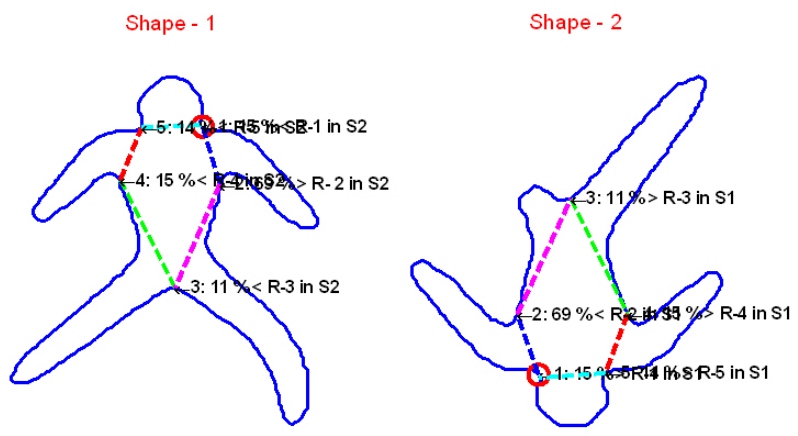

Figure 4. RMA applied to two shapes of "dude" category from Kimia database for local region matching. Regions are identified accurately in this example while quantifying regional change in each part. The regions are identified correctly because of the prior initial alignment, which is basically to align shape signatures to start from the most similar points.

The relatively poor results of RMA on hand shapes stem from the fact that the inner left, right and center fingers of each hand have very similar structures. Similarly, the symmetry of kite shapes is also a misleading factor in identifying regions correctly. In machine vision, matching asymmetry is generally accepted and may not be counted as an error, in which case the results improve dramatically. Incorporating translational and reflection symmetries into our framework, while enabling the detection of new growths and occlusions; is the subject of current work.
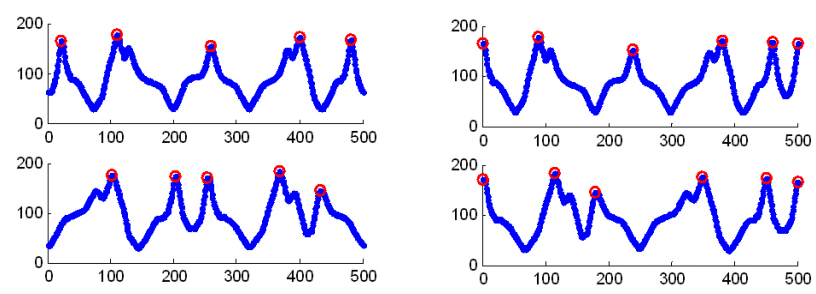

Figure 5. Integral invariant signature at the coarsest scale with causal peaks of two shapes in Figure 4, for shape-1 on the top and shape- 2 in the bottom row. Left: the signatures are shown without initial alignment. Right: after initial alignment; the causal peaks at the coarsest scale are highlighted in red. Plots of $I_{\gamma}^{r}(p)$, against the parameterization of the shape at every point $p$.
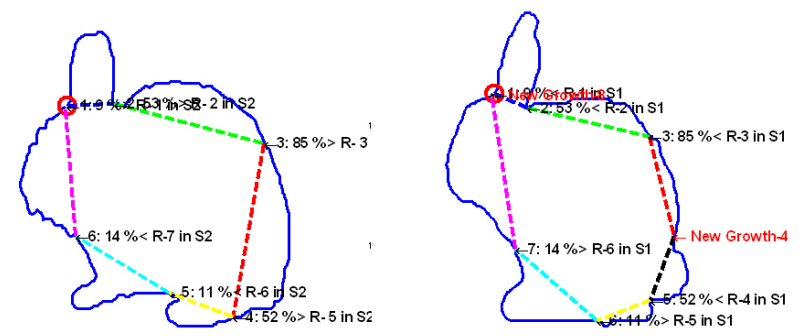

Figure 6. RMA applied to two shapes of "bunny" category from Kimia database for local region matching. Shape on the right is been identified with a new growth in comparison with the shape on the left.

The temporal mammograms that were used in this study were made available to us by Matakina Technologies. We begin by applying a variation of the hierarchical algorithm based on iso-contours. ${ }^{[131]}$ The reason for using this algorithm here is that it is computationally very efficient, and indeed it can be the basis of a real time system, even without resorting to a GPU implementation. The algorithm segments the complete internal topography of the breast in a structured way that can subsequently be used to establish correspondences between mammograms. The algorithm has worked well of mammograms we have processed to date. 
Table 1. Results of applying RMA to variable and occluded shapes from the Kimia database. Note that matching asymmetry is considered to be an error (we have mammograms in mind), though in machine vision applications it may be deemed acceptable, which would further improve the results.

\begin{tabular}{llllll}
\hline Criteria & “bunny”/Rabbits & “dude”/Man & “fgen”/Alien & “kk”/Kite & “hand” \\
\hline Initial Alignment & 91.7 & 100 & 91.67 & 77.78 & 55.56 \\
Detection of new growth & 98.05 & None & 90 & 91.38 & 53.34 \\
Regional Correspondence & 98.33 & 100 & 86.11 & 91.61 & 78.05 \\
\hline
\end{tabular}
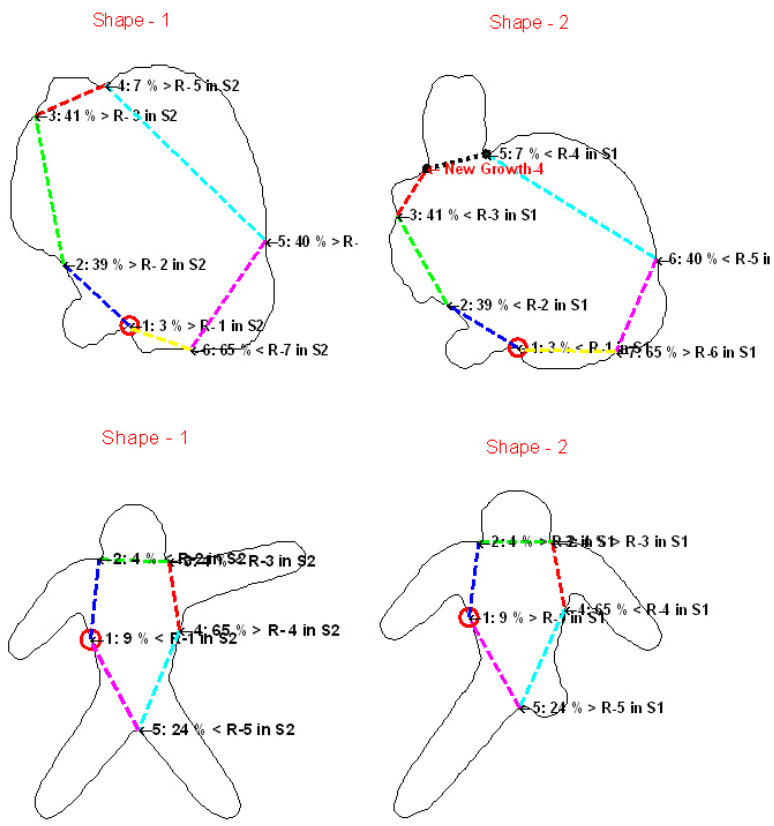

Figure 7. Continued to the next page for caption

An example of alignment for a segmentation of nested regions is shown in Figure 9. RMA matches regions in shapes irrespective of their sequence. However, in this particular case the accuracy of matching is obvious by comparing the II signatures of both mammograms after initial key-point alignment.

Shape correspondence using RMA has been applied to the regions segmented in this way, as illustrated in Figure 10. The mammograms are de-noised using a Perona-Malik anisotropic diffusion filter. The lesions from pairs of temporal mammograms are put into regional correspondences. In some cases the algorithm identifies the segments (and associated sub-regions) that correspond to new growth, while at the same time calculating the percentage change in other sub-regions. It may be noted that the number of regions in both shapes may not be equal. Some obvious mismatches can also be seen where the regional differences are substantial or the non-corresponding regions are very similar. The correspondence of regions does not currently depend upon the texture or gradient information enclosed in them. DePublished by Sciedu Press tailed examples of RMA applied to regions segmented and matched from temporal mammograms are given in Figure 11.
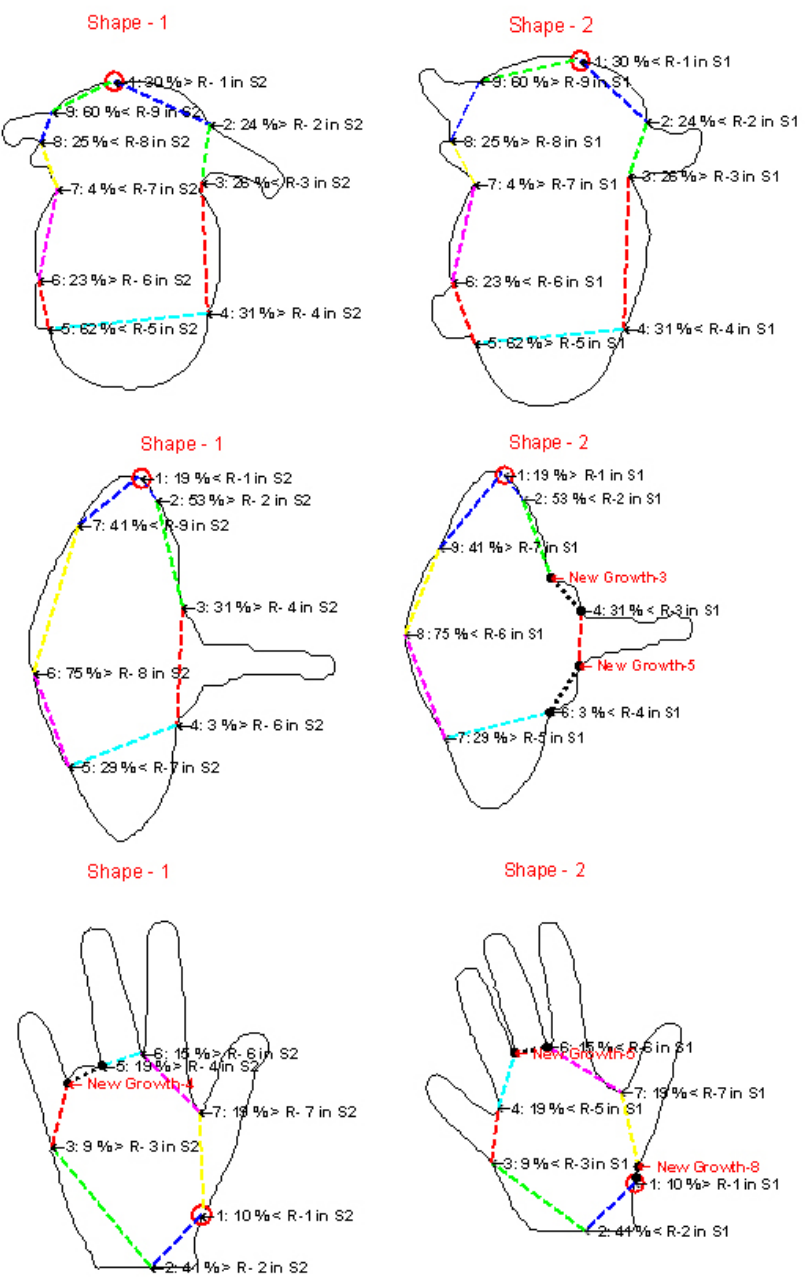

Figure 8. Examples of RMA application on 2D planar shapes of varying spatial scale. Some mismatches can also be seen as the algorithm is similarity driven, independent of spatial information.

\section{Conclusion}

This paper has introduced a novel local RMA using II scale space. Shape matching and correspondence algorithms usu- 
ally match and establish point-wise correspondences between two shapes and may even handle partial occlusion. However, they typically do not quantify partial occlusions nor identify complete occlusions or new growth. It is important to measure regional differences quantitatively within each shape and establish correspondences based upon region matching. For masses, it is vital to analyze their growth and notice the emergence or disappearance of any region. This can be helpful in detecting new growths and identifying their orientation. Following region of interest segmentation, we have introduced a method of local shape correspondence and region matching using II scale space. II are calculated for segmented shapes from mammograms at all scales. The algorithm identifies causal peaks of this scale space as key points and breaks the shape into sub-regions based upon them. The best matching region is selected as a point of initial alignment and regions are corresponded based on a similarity measure. RMA gives encouraging results in detecting tumor growths and its aggressiveness with respect to shape.
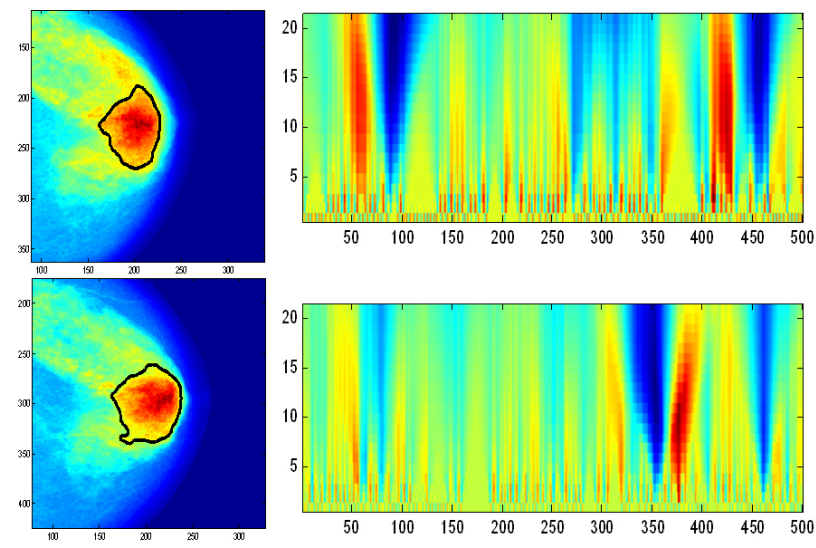

Figure 9. Segmented regions from temporal mammograms on the left while their corresponding scale spaces is given on the right hand side

The fundamental limitation of this approach is that it does not know the spatial layout of the regions it is matching, which of course has to be spatially invariant. This has resulted in the mismatching in Figures 7, 8 and 12, and will not improve existing models if this framework is extended to the point-wise corresponding. Transformations to incorporate information from inside of the shape can be applied prior to applying RMA, which will add value to the shape signature and reduce correspondence error. Some of the examples of such shape transformations are bending invariants, eccentricity transform and interpolation of medial axis within the shape.

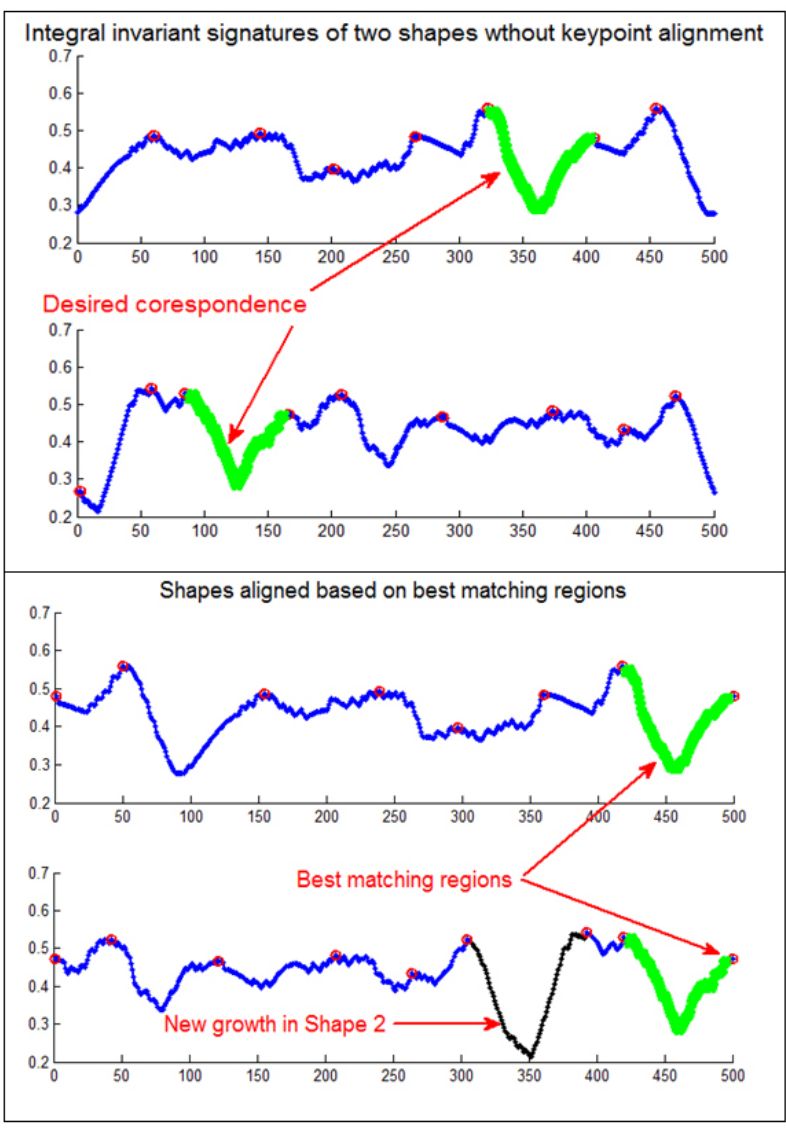

Figure 10. Above is the scale space signature of two corresponding shapes in Figure 8 at the coarsest scale. On the left, the signatures are unaligned whereas on the right side they are aligned using RMA.
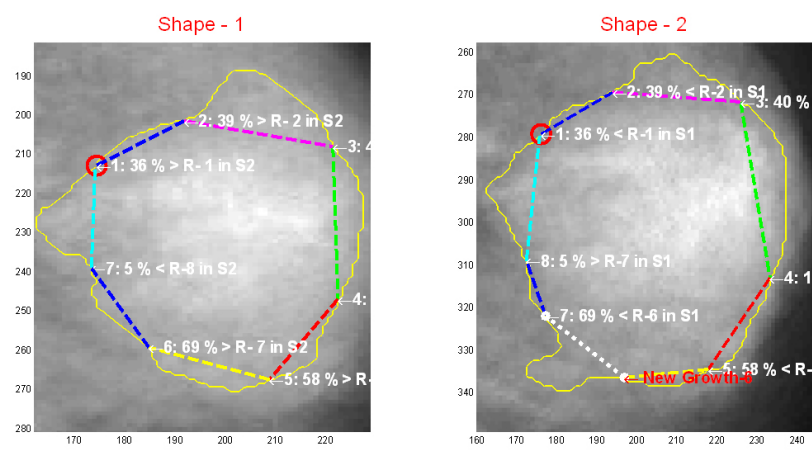

Figure 11. Final RMA results of the regions segmented in Figure 9 and aligned for a match in Figure 10. Detailed examples are given in Figure 12.

\section{Conflicts of InTERest Disclosure}

The authors declare that they have no competing interests. 

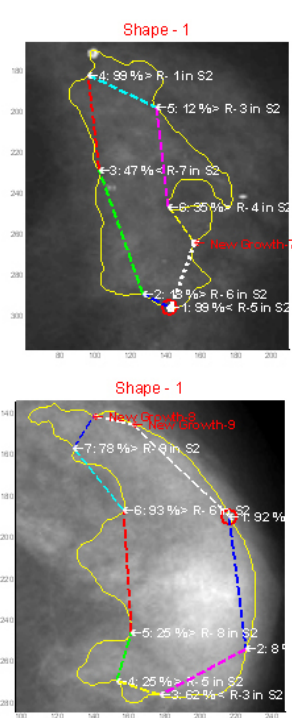

Shape - 1

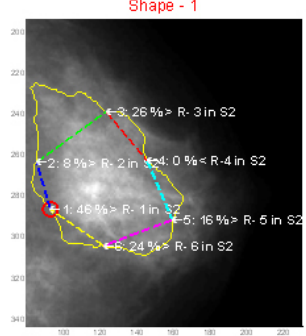

Shape - 1

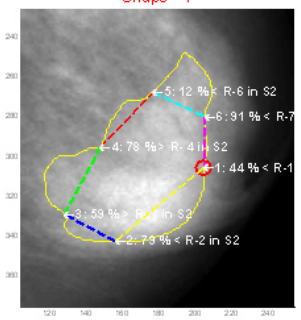

Shape - 1

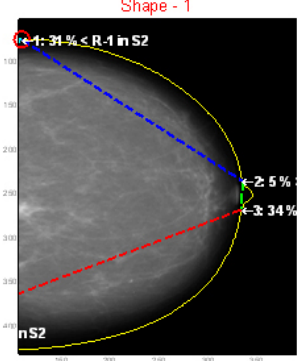

Shape - 1

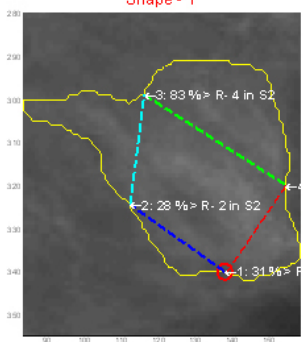

Shape - 2

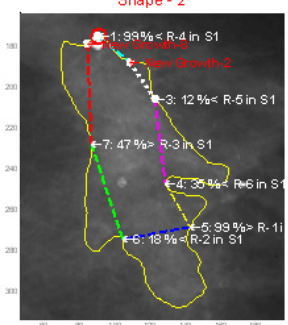

Shape - 2

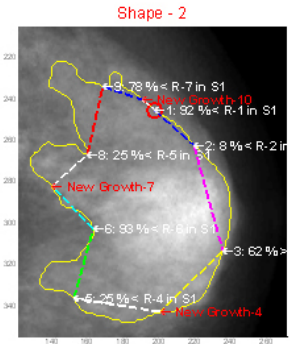

Shape - 2

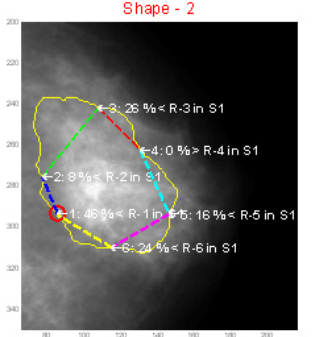

Shape - 2

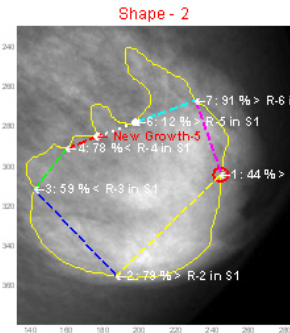

Shape - 2

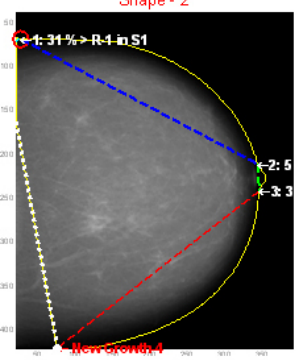

Shape - 2

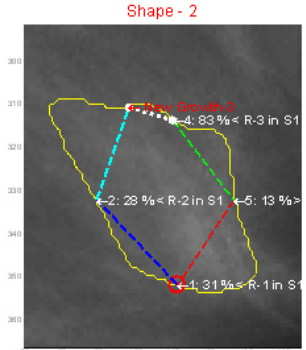

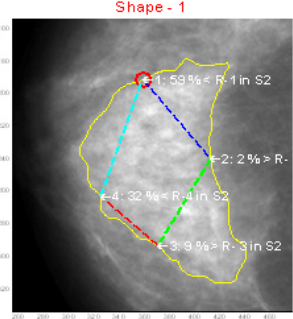

Shape - 1

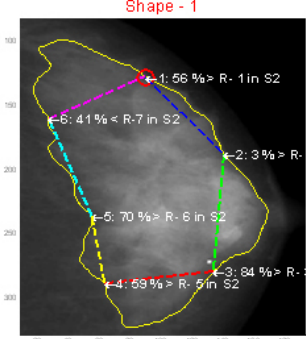

Shape - 1
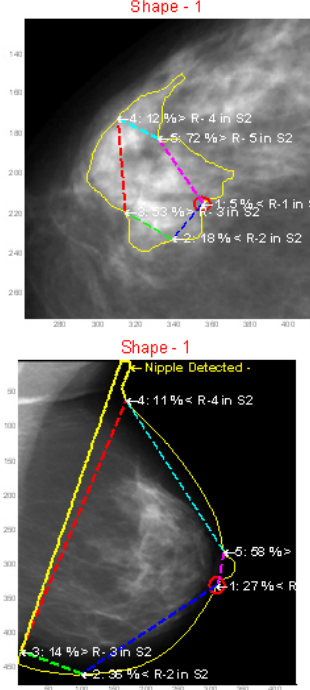

Shape - 1
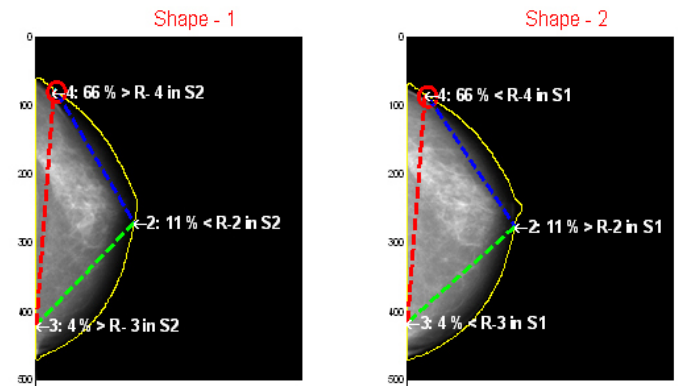

Figure 12. Region matching of corresponding contours on the temporal mammograms. The red circles in the shapes identify points of initial alignment. Regions are color-coded and show both good and bad examples of regional correspondences. 


\section{REFERENCES}

[1] Zhang S, Ma KK. A novel shape matching method using biological sequence Dynamic alignment. Multimedia and Expo. 2000. ICME 2000. 2000 IEEE International Conference. 2000; 1: 343-346.

[2] Yu B, Guo L, Zhao T, et al. A curve matching algorithm based on Freeman Chain Code. Intelligent Computing and Intelligent Systems (ICIS), 2010 IEEE International Conference. 2010; 3: 669-672.

[3] Kendall DG. Shape manifolds, procrustean metrics, and complex projective spaces. Bull. Lond. Math. Soc. 1984; 16: 81-121. http: //dx.doi.org/10.1112/blms/16.2.81

[4] Duci A, Yezzi AJ, Mitter SK, et al. Shape representation via harmonic embedding. Computer Vision, 2003. Proceedings. Ninth IEEE International Conference. 2003; 656-662. http://dx. doi .org/1 0.1109/ICCV. 2003.1238410

[5] Sharon E, Mumford D. 2d-shape analysis using conformal mapping. Int. J. Comput. Vis. 2006; 70: 55-75. http://dx. doi .org/10.10 07/s11263-006-6121-z

[6] Zhang D, Lu G. Review of shape representation and description techniques. Pattern Recognit. 2004; 37: 1-19. http://dx .doi .org/1 $0.1016 / j$.patcog. 2003.07 .008

[7] Mumford D. Mathematical theories of shape: Do they model perception? San Diego,'91, San Diego, CA. 1991; 2-10.

[8] Mardia KV, Dryden IL. Shape distributions for landmark data. Adv. Appl. Probab. 1989; 742-755. http://dx.doi.org/10.2307/1 427764

[9] Amanatiadis A, Kaburlasos VG, Gasteratos A, et al. Evaluation of shape descriptors for shape-based image retrieval. Image Process. IET. 2011; 5: 493-499. http://dx.doi.org/10.1049/iet-ipr .2009 .0246

[10] Gu YH, Tjahjadi T. Coarse-to-fine planar object identification using invariant curve features and B-spline modeling. Pattern Recognit. 2000; 33: 1411-1422. http://dx.doi.org/10.1016/S0031-3 203(99) 00131-4

[11] Cohen FS, Wang JY. Part I: Modeling image curves using invariant 3-D object curve models-a path to 3-D recognition and shape estimation from image contours. Pattern Anal. Mach. Intell. IEEE Trans. 1994; 16: 1-12. http://dx.doi.org/10.1109/34.273721

[12] Huang Z, Cohen FS. Affine-invariant B-spline moments for curve matching. Image Process. IEEE Trans. 1996; 5: 1473-1480. PMid: 18290064. http://dx.doi.org/10.1109/83.536895

[13] Wang Y, Teoh EK, Shen D. Structure-adaptive B-snake for segmenting complex objects. Image Processing. 2001 International Conference. $2001 ; 2$ : 769-772. PMid: 11314010.

[14] Wang Y, Teoh EK, Shen D. Lane detection and tracking using BSnake. Image Vis. Comput. 2004; 22: 269-280. http://dx.doi.o $\mathrm{rg} / 10.1016 / \mathrm{j}$.imavis.2003.10.003

[15] Wang Y, Teoh EK. A novel 2D shape matching algorithm based on B-spline modeling. Image Processing, 2004. ICIP’04. 2004 International Conference. 2004; 1: 409-412.

[16] Arrebola F, Sandoval F. Corner detection and curve segmentation by multiresolution chain-code linking. Pattern Recognit. 2005; 38: 1596 1614. http://dx.doi.org/10.1016/j.patcog.2005.03.006

[17] Mokhtarian F, Mackworth A. Scale-based description and recognition of planar curves and two-dimensional shapes. Pattern Anal. Mach. Intell. IEEE Trans. 1986; 34-43.

[18] Mokhtarian F, Abbasi S, Kittler J. Efficient and robust retrieval by shape content through curvature scale space. Ser. Softw. Eng. Knowl. Eng. 1997; 8: 51-58.

[19] Thomas TY. The differential invariants of generalized spaces. Cambridge. $1934 ; 75$.
[20] Olver PJ. Equivalence, invariants and symmetry. UK: Cambridge University Press; 1995. http://dx.doi .org/10.1017/CB09780 511609565

[21] Manay S, Hong BW, Yezzi AJ, et al. Integral invariant signatures. Springer. 2004. http://dx . doi .org/10.1007/978-3-540-246 73-2_8

[22] Huang QX, Flöry S, Gelfand N, et al. Reassembling fractured objects by geometric matching. ACM Transactions on Graphics (TOG). 2006; 25: 569-578. http://dx.doi.org/10.1145/1141911.1141925

[23] Yang YL, Lai YK, Hu SM, et al. Robust principal curvatures on multiple scales. Symposium on Geometry Processing. 2006; 223-226.

[24] Manay S, Cremers D, Hong BW, et al. Integral invariants for shape matching. Pattern Anal. Mach. Intell. IEEE Trans. 2006; 28: $1602-$ 1618. PMid: 16986542. http://dx.doi.org/10.1109/TPAMI. 2 006.208

[25] Al-Kadi OS. Assessment of texture measures susceptibility to noise in conventional and contrast enhanced computed tomography lung tumour images. Comput. Med. Imaging Graph. 2010; 34: 494-503. PMid: 20060263. http://dx.doi.org/10.1016/j.compmedim ag. 2009.12.011

[26] Al-Kadi OS, Watson D. Susceptibility of texture measures to noise: an application to lung tumor $\mathrm{CT}$ images. BioInformatics and BioEngineering. 2008. BIBE 2008. 8th IEEE International Conference. 2008; 1-4. http://dx.doi.org/10.1109/bibe.2008.4696789

[27] Al-Kadi OS. Tumour grading and discrimination based on class assignment and quantitative texture analysis techniques [Dissertation] [Brighton]: University of Sussex; 2009. http://dx.doi .org/10. 13140/RG.2.1.1930.0004

[28] Sato J, Cipolla R. Affine integral invariants for extracting symmetry axes. Image Vis. Comput. 1997; 15: 627-635. http://dx.doi.o $\mathrm{rg} / 10.1016 / \mathrm{S} 0262-8856$ (97)00011-5

[29] Sato J, Cipolla R. Affine integral invariants and matching of curves. Pattern Recognition. 1996, Proceedings of the 13th International Conference. 1996; 1: 915-919. http://dx.doi.org/10.1109/i cpr. 1996.546157

[30] Bauer M, Fidler T, Grasmair M. Local uniqueness of the circular integral invariant. arXiv Prepr. arXiv:1107.4257. 2011.

[31] Gdalyahu Y, Weinshall D. Flexible syntactic matching of curves and its application to automatic hierarchical classification of silhouettes. Pattern Anal. Mach. Intell. IEEE Trans. 1999; 21: 1312-1328. http://dx.doi.org/10.1109/34.817410

[32] Petrakis EGM, Diplaros A, Milios E. Matching and retrieval of distorted and occluded shapes using dynamic programming. Pattern Anal. Mach. Intell. IEEE Trans. 2002; 24: 1501-1516. http: //dx.doi.org/10.1109/TPAMI. 2002.1046166

[33] Davis LS. Understanding shape: Angles and sides. Comput. IEEE Trans. 1977; 100: 236-242. http://dx.doi.org/10.1109/TC. 1977.1674812

[34] Ozcan E, Mohan CK. Partial shape matching using genetic algorithms. Pattern Recognit. Lett. 1997; 18: 987-992. http://dx.doi . org/10.1016/S0167-8655(97)00123-2

[35] Ion A, Peyré G, Haxhimusa Y, et al. Shape matching using the geodesic eccentricity transform-a study. Proceedings of the 31st annual workshop of the Austrian Association for Pattern (OAGM/AAPR). 2007; 97-104.

[36] Belongie S, Malik J, Puzicha J. Shape matching and object recognition using shape contexts. Pattern Anal. Mach. Intell. IEEE Trans. 2002; 24: 509-522. http://dx.doi.org/10.1109/34.993558

[37] Rusinol M, Dosch P, Lladós J. Boundary shape recognition using accumulated length and angle information. Pattern Recognition and Image Analysis. Springer. 2007; 210-217. 
[38] Mokhtarian F, Mackworth AK. A theory of multiscale, curvaturebased shape representation for planar curves. IEEE Trans. Pattern Anal. Mach. Intell. 1992; 14: 789-805. http://dx.doi.org/10. $1109 / 34.149591$

[39] Zahn CT, Roskies RZ. Fourier descriptors for plane closed curves. Comput. IEEE Trans. 1972; 100: 269-281. http://dx.doi.org/1 $0.1109 /$ TC. 1972.5008949

[40] Sharma A, Horaud R. Shape matching based on diffusion embedding and on mutual isometric consistency. Computer Vision and Pattern Recognition Workshops (CVPRW), 2010 IEEE Computer Society Conference. 2010; 29-36. http://dx.doi.org/10.1109/cvprw .2010 .5543278

[41] Bronstein AM, Bronstein MM, Kimmel R. Generalized multidimensional scaling: a framework for isometry-invariant partial surface matching. Proc. Natl. Acad. Sci. United States Am. 2006; 103: 11681172. PMid: 16432211. http://dx.doi.org/10.1073/pnas. 05 08601103

[42] Mateus D, Horaud R, Knossow D, et al. Articulated shape matching using Laplacian eigenfunctions and unsupervised point registration. Computer Vision and Pattern Recognition. 2008. CVPR 2008. IEEE Conference. 2008; 1-8. http://dx.doi.org/10.1109/cvpr. 20 08.4587538

[43] Ruggeri MR, Patanè G, Spagnuolo M, et al. Spectral-driven isometryinvariant matching of 3D shapes. Int. J. Comput. Vis. 2010; 89: 248-265. http://dx.doi.org/10.1007/s11263-009-0250-0

[44] Wang S, Wang Y, Jin M, et al. Conformal geometry and its applications on 3d shape matching, recognition, and stitching. Pattern Anal. Mach. Intell. IEEE Trans. 2007; 29: 1209-1220. PMid: 17496378. http://dx.doi.org/10.1109/TPAMI .2007.1050

[45] Bronstein AM, Bronstein MM, Bruckstein AM, et al. Analysis of two-dimensional non-rigid shapes. Int. J. Comput. Vis. 2008; 78: 67-88. http://dx.doi.org/10.1007/s11263-007-0078-4

[46] Hamza AB, Krim H. Geodesic matching of triangulated surfaces. Image Process. IEEE Trans. 2006; 15: 2249-2258. http://dx. doi .org/10.1109/TIP. 2006.875250

[47] Osada R, Funkhouser T, Chazelle B, et al. Shape distributions. ACM Trans. Graph. 2002; 21: 807-832. http://dx.doi.org/10.1145 $/ 571647.571648$

[48] Ling H, Jacobs DW. Shape classification using the inner-distance. Pattern Anal. Mach. Intell. IEEE Trans. 2007; 29: 286-299. PMid: 17170481. http://dx.doi.org/10.1109/TPAMI. 2007.41

[49] Nasreddine K, Benzinou A, Fablet R. Shape geodesics for boundarybased object recognition and retrieval. Image Processing (ICIP), 2009 16th IEEE International Conference. 2009; 405-408. http: //dx.doi.org/10.1109/icip. 2009.5414454

[50] Siddiqi K, Shokoufandeh A, Dickinson SJ, et al. Shock graphs and shape matching. Int. J. Comput. Vis. 1999; 35: 13-32. http: //dx.doi.org/10.1023/A:1008102926703

[51] Leordeanu M, Hebert M. A spectral technique for correspondence problems using pairwise constraints. Computer Vision, 2005. ICCV 2005. Tenth IEEE International Conference. 2005; 2: 1482-1489. http://dx.doi.org/10.1109/iccv.2005.20

[52] Duchenne O, Bach F, Kweon IS, et al. A tensor-based algorithm for high-order graph matching. Pattern Anal. Mach. Intell. IEEE Trans. 2011; 33: 2383-2395. PMid: 21646677. http://dx.doi.org/10. 1109/TPAMI . 2011. 110

[53] Torresani L, Kolmogorov V, Rother C. Feature correspondence via graph matching: Models and global optimization. Computer Vision-ECCV 2008, Springer. 2008; 596-609.

[54] Maciel J, Costeira JP. A global solution to sparse correspondence problems. Pattern Anal. Mach. Intell. IEEE Trans. 2003; 25: 187-199. http://dx.doi.org/10.1109/TPAMI .2003.1177151
[55] Xu C, Liu J, Tang X. 2D shape matching by contour flexibility. Pattern Anal. Mach. Intell. IEEE Trans. 2009; 31: 180-186. PMid: 19029556. http://dx.doi.org/10.1109/TPAMI . 2008.199

[56] Reuter M, Wolter FE, Peinecke N. Laplace-spectra as fingerprints for shape matching. Proceedings of the 2005 ACM symposium on Solid and physical modeling. 2005; 101-106. http://dx.doi .org/10. 1145/1060244.1060256

[57] Gorelick L, Galun M, Sharon E, et al. Shape representation and classification using the poisson equation. Pattern Anal. Mach. Intell. IEEE Trans. 2006; 28: 1991-2005. PMid: 17108372. http: //dx.doi.org/10.1109/TPAMI. 2006.253

[58] Chen YW, Xu CL. Rolling penetrate descriptor for shape-based image retrieval and object recognition. Pattern Recognit. Lett. 2009; 30: 799804. http://dx.doi.org/10.1016/j. patrec. 2008.04.015

[59] Sebastian TB, Klein PN, Kimia BB. On aligning curves. Pattern Anal. Mach. Intell. IEEE Trans. 2003; 25: 116-125. http://dx.doi.o $\mathrm{rg} / 10.1109 /$ TPAMI .2003 .1159951

[60] Latecki LJ, Lakamper R, Eckhardt T. Shape descriptors for non-rigid shapes with a single closed contour. Computer Vision and Pattern Recognition, 2000. Proceedings. IEEE Conference. 2000; 1: 424-429. http://dx.doi.org/10.1109/cvpr.2000.855850

[61] Belongie S, Malik J, Puzicha J. Matching shapes. Computer Vision, 2001. ICCV 2001. Proceedings. Eighth IEEE International Conference. 2001; 1: 454-461. http://dx. doi .org/10.1109/iccv. 20 01.937552

[62] Janan F, Brady M. Shape matching by integral invariants on eccentricity transformed images. Engineering in Medicine and Biology Society (EMBC), 2013 35th Annual International Conference of the IEEE. 2013; 5099-5102. http://dx.doi.org/10.1109/emb c. 2013.6610695

[63] Sethian JA. Level set methods and fast marching methods: evolving interfaces in computational geometry, fluid mechanics, computer vision, and materials science. UK: Cambridge university press; 1999 Vol. 3.

[64] Kimmel R. Fast Marching Methods. Numerical Geometry of Images. Springer. 2004; 87-108. http://dx.doi.org/10.1007/9 78-0-387-21637-9_7

[65] Frenkel M, Basri R. Curve matching using the fast marching method Energy Minimization Methods in Computer Vision and Pattern Recognition. 2003; 35-51. http://dx. doi .org/10 . 1007/978-3 -540-45063-4_3

[66] Bildverarbeitung AM, Ion DIA. The Eccentricity Transform of nDimensional Shapes with and without Boundary.

[67] Ion A, Artner NM, Peyré G, et al. Matching 2D and 3D articulated shapes using the eccentricity transform. Comput. Vis. Image Underst. 2011; 115: 817-834. http://dx.doi.org/10.1016/j.cviu. 20 11.02 .006

[68] Ion A, Artner NM, Peyré G, et al. 3D shape matching by geodesic eccentricity. Computer Vision and Pattern Recognition Workshops, 2008. CVPRW'08. IEEE Computer Society Conference. 2008; 1-8.

[69] Sundar H, Silver D, Gagvani N, et al. Skeleton based shape matching and retrieval. Shape Modeling International, 2003. 2003; 130-139.

[70] Xu Y, Wang B, Liu W, et al. Skeleton graph matching based on critical points using path similarity. Computer Vision-ACCV 2009, Springer. 2010; 456-465.

[71] Zhao $\mathrm{H}$. A fast sweeping method for eikonal equations. Math. Comput. 2005; 74: 603-627. http://dx.doi .org/10.1090/S0025-5 718-04-01678-3

[72] Tsai YHR, Cheng LT, Osher S, et al. Fast sweeping algorithms for a class of Hamilton-Jacobi equations. SIAM J. Numer. Anal. 2003; 41: 673-694. http://dx.doi.org/10.1137/S0036142901396533 
[73] Tian J, Ma L, Yu W. Ant colony optimization for wavelet-based image interpolation using a three-component exponential mixture model. Expert Syst. with Appl. 2011; 38: 12514-12520. http: //dx.doi.org/10.1016/j.eswa.2011.04.037

[74] Teodorovic D, Davidovic T, Selmic M. Bee colony optimization: the applications survey. ACM Trans. Comput. Log. 2011; 1529.

[75] Davidovic T, Ramljak D, Selmic M, et al. Parallel bee colony optimization for scheduling independent tasks on identical machines. Proceedings of the International Symposium on Operational Research. 2010; 389-392.

[76] Xu C, Duan H. Artificial bee colony (ABC) optimized edge potential function (EPF) approach to target recognition for low-altitude aircraft. Pattern Recognit. Lett. 2010; 31: 1759-1772. http://dx.doi .org /10.1016/j.patrec. 2009.11.018

[77] Elad A, Kimmel R. On bending invariant signatures for surfaces. Pattern Anal. Mach. Intell. IEEE Trans. 2003; 25: 1285-1295. http://dx.doi.org/10.1109/TPAMI . 2003.1233902

[78] Rosin PL. Shape description by bending invariant moments. Computer Analysis of Images and Patterns. 2011; 253-260. http://dx .doi.org/10.1007/978-3-642-23672-3_31

[79] Veltkamp RC, Hagedoorn M. State of the art in shape matching. Springer. 2001. http://dx.doi.org/10.1007/978-1-4471-3 702-3_4

[80] Mikolajczyk K, Schmid C. A performance evaluation of local descriptors. Pattern Anal. Mach. Intell. IEEE Trans. 2005; 27: 1615-1630. PMid: 16237996. http://dx.doi.org/10.1109/TPAMI. 2005 188

[81] Kaick OV, Zhang H, Hamarneh G, et al. A survey on shape correspondence. Computer Graphics Forum. 2011; 30: 1681-1707. http://dx.doi.org/10.1111/j.1467-8659.2011.01884.x

[82] Sonka M, Hlavac V, Boyle R. Image processing, analysis, and machine vision. 1999.

[83] Li SZ. Shape matching based on invariants. 1998.

[84] Mumford D, Latto A, Shah J. The representation of shape. Proc. IEEE Workshop Computer Vision. 1984; 183-191.

[85] Reiss TH. Recognizing planar objects using invariant image features. Springer-Verlag New York, Inc. 1993. http://dx.doi.org/10. $1007 / \mathrm{BFb} 0017553$

[86] Chetverikov D, Khenokh Y. Matching for shape defect detection. Computer Analysis of Images and Patterns. 1999; 367-374. http: //dx.doi.org/10.1007/3-540-48375-6_44

[87] Alferez R, Wang YF. Geometric and illumination invariants for object recognition. Pattern Anal. Mach. Intell. IEEE Trans. 1999; 21 : 505-536. http://dx.doi.org/10.1109/34.771318

[88] Bengtsson A, Eklundh JO. Shape representation by multiscale contour approximation. IEEE Trans. Pattern Anal. Mach. Intell. 1991; 13: 85-93. http://dx.doi.org/10.1109/34.67634

[89] Brandt RD, Lin F. Representations that uniquely characterize images modulo translation, rotation, and scaling. Pattern Recognit. Lett. 1996; 17: 1001-1015. http://dx.doi.org/10.1016/0167-865 5 (96) 00062-1

[90] Bruckstein AM, Rivlin E, Weiss I. Scale space semi-local invariants. Image Vis. Comput. 1997; 15: 335-344. http://dx.doi.org/10. 1016/S0262-8856(96) 01140-7

[91] Cohignac T, Lopez C, Morel JM. Integral and local affine invariant parameter and application to shape recognition. Pattern Recognition. 1994. Vol. 1-Conference A: Computer Vision \& Image Processing., Proceedings of the 12th IAPR International Conference. 1994; 1: 164-168. http://dx.doi.org/10.1109/icpr.1994.576250

[92] Helgason S. Groups \& Geometric Analysis: Radon Transforms, Invariant Differential Operators and Spherical Functions. Access Online via Elsevier. vol. 1. 1984.
[93] Squire DM, Caelli TM. Invariance Signatures: Characterizing contours by their departures from invariance. Comput. Vis. Image Underst. 2000; 77: 284-316. http://dx.doi.org/10.1006/cviu. 2000.0809

[94] Forsyth D, Mundy JL, Zisserman A, et al. Projectively invariant representations using implicit algebraic curves. Springer. 1990. http://dx.doi.org/10.1007/bfb0014893

[95] Nielsen L, Sparr G. Projective area-invariants as an extension of the cross-ratio. CVGIP: Image Underst. 1991; 54: 145-159. http: //dx.doi.org/10.1016/1049-9660(91)90079-5

[96] Huang CL, Huang DH. A content-based image retrieval system. Image Vis. Comput. 1998; 16: 149-163. http://dx.doi.org/10.10 16/S0262-8856(97)00062-0

[97] Rothwell CA, Zisserman A, Forsyth DA, et al. Canonical frames for planar object recognition. Computer Vision-ECCV'92. 1992; 757-772. http://dx.doi.org/10.1007/3-540-55426-2_86

[98] Rothwell CA, Zisserman A, Forsyth DA, et al. Planar object recognition using projective shape representation. Int. J. Comput. Vis. 1995; 16: 57-99. http://dx.doi.org/10.1007/BF01428193

[99] Shashua A, Navab N. Relative affine structure: Canonical model for 3D from 2D geometry and applications. Pattern Anal. Mach. Intell IEEE Trans. 1996; 18: 873-883. http://dx.doi.org/10.1109 /34. 537342

[100] Zisserman A, Forsyth D, Mundy J, et al. 3D object recognition using invariance. Artif. Intell. 1995; 78: 239-288. http://dx.doi.org /10.1016/0004-3702(95)00023-2

[101] Gool LV, Moons T, Ungureanu D. Affine/photometric invariants for planar intensity patterns. Computer Vision-ECCV'96, Springer. 1996; 642-651. http://dx.doi.org/10.1007/BFb0015574

[102] Forsyth D, Mundy JL, Zisserman A, et al. Invariant descriptors for 3 d object recognition and pose. IEEE Trans. Pattern Anal. Mach. Intell. 1991; 13: 971-991. http://dx.doi.org/10.1109/34.99233

[103] Lasenby J, Bayro-Corrochano E, Lasenby AN, et al. A new framework for the formation of invariants and multiple-view constraints in computer vision. Image Processing, 1996. Proceedings., International Conference. 1996; 1: 313-316. http://dx.doi.org/10.1109/i cip. 1996.560819

[104] Cole JB, Murase H, Naito S. A Lie group theoretic approach to the invariance problem in feature extraction and object recognition. Pattern Recognit. Lett. 1991; 12: 519-523. http://dx.doi.org/10. 1016/0167-8655 (91)90091-Y

[105] Calabi E, Olver PJ, Shakiban C, et al. Differential and numerically invariant signature curves applied to object recognition. Int. J. Comput. Vis. 1998; 26: 107-135. http://dx.doi.org/10.1023/A: 1007992709392

[106] Kanatani K. Group-theoretical methods in image understanding. Springer-Verlag Berlin, vol. 2. 1990. http://dx.doi .org/10.10 07/978-3-642-61275-6

[107] Lenz R. Group theoretical methods in image processing. SpringerVerlag New York, Inc. 1990. http://dx.doi.org/10.1007/3-5 40-52290-5

[108] Trucco E. Geometric Invariance in Computer Vision. AI Commun. 1995; 8: 193-194.

[109] Cao W, Hu P, Liu Y, et al. Gaussian-curvature-derived invariants for isometry. Sci. China Inf. Sci. 2011; 1-12.

[110] White R, Kamath C, Newsam S. Matching Shapes Using Local Descriptors. United States. Department of Energy. 2004. http: //dx.doi.org/10.2172/15014679

[111] Taubin G, Cooper DB. Object recognition based on moment (or algebraic) invariants. IBM TJ Watson Research Center. 1991.

[112] Fidler T, Grasmair M, Pottmann H, et al. Inverse problems of integral invariants and shape signatures. Preprint. 2007. 
[113] Smith SM, Brady JM. SUSAN-A new approach to low level image processing. Int. J. Comput. Vis. 1997; 23: 45-78. http: //dx.doi.org/10.1016/S0042-6989(96)00147-2

[114] Mansoory MS, Ashtiyani M, Sarabadani H. Automatic Crack Detection in Eggshell Based on SUSAN Edge Detector Using Fuzzy Thresholding. Mod. Appl. Sci. 2011; 5.

[115] Arun KS, Sarath KS. Evaluation of SUSAN Filter for the Identification of Micro Calcification. Int. J. Comput. Appl. 2011; 15: 41-44.

[116] LI Y, GAO Y, LU L, et al. Hybrid corner detection algorithm for brain magnetic resonance image registration. Chin. J. Med. Imaging Technol. 2012; 2.

[117] Si-ming H, Bing-han L, Wei-zhi W. Moving shadow detection based on Susan algorithm. Computer Science and Automation Engineering (CSAE), 2011 IEEE International Conference. 2011; 3: 16-20. http://dx.doi.org/10.1109/CSAE. 2011.5952625

[118] Xu S, Han L, Zhang L. An Algorithm to Edge Detection Based on SUSAN Filter and Embedded Confidence. Intelligent Systems Design and Applications, 2006. ISDA'06. Sixth International Conference. 2006; 2: 720-723. http://dx.doi.org/10.1109/isda. 2006.253700

[119] Rezai-Rad G, Aghababaie M. Comparison of SUSAN and sobel edge detection in MRI images for feature extraction. Information and Communication Technologies, 2006. ICTTA'06. 2nd. 2006; 1 : 1103-1107.

[120] Zeng J, Li D. SUSAN edge detection method for color image. Jisuanji Gongcheng yu Yingyong. 2011; 47: 194-196.

[121] Qu ZG, Wang P, Gao YH, et al. Randomized SUSAN edge detector. Opt. Eng. 2011; 50: 110502-110502. http://dx.doi.org/10.11 $17 / 1.3647520$

[122] Rafajlowicz E. SUSAN edge detector reinterpreted, simplified and modified. Multidimensional (nD) Systems, 2007 International Work- shop. 2007.69-74. http://dx.doi.org/10.1109/nds.2007.45 09548

[123] Xingfang Y, Yumei H, Yan L. An improved SUSAN corner detection algorithm based on adaptive threshold. Signal Processing Systems (ICSPS), 2010 2nd International Conference. 2010; 2. http://dx.doi.org/10.1109/icsps.2010.5555730

[124] Kliot M, Rivlin E. Invariant-based shape retrieval in pictorial databases. Computer Vision-ECCV'98, Springer. 1998; 491-507. http://dx.doi.org/10.1007/BFb0055686

[125] Davies ER. Machine vision: theory, algorithms, practicalities. Access Online via Elsevier. 2004.

[126] Janan F, Brady SM. Integral invariants for image enhancement. Engineering in Medicine and Biology Society (EMBC), 2013 35th Annual International Conference of the IEEE. 2013; 4018-4021. http://dx.doi.org/10.1109/embc. 2013.6610426

[127] Janan F, Brady M. Region matching in the temporal study of mammograms using integral invariant scale-space. Breast Imaging, Springer. 2012; 173-180.

[128] Weiss I. Noise-resistant invariants of curves. Pattern Anal. Mach. Intell. IEEE Trans. 1993; 15: 943-948. http://dx.doi.org/10. $1109 / 34.232081$

[129] Hann CA, Hickman MS. Projective curvature and integral invariants. Acta Appl. Math. 2002; 74: 177-193. http://dx.doi .org/10.10 23/A : 1020617228313

[130] Pottmann H, Wallner J, Huang QX, et al. Integral invariants for robust geometry processing. Comput. Aided Geom. Des. 2009; 26 37-60. http://dx.doi.org/10.1016/j.cagd.2008.01.002

[131] Hong BW, Brady M. Segmentation of mammograms in topographic approach. 2003. 